\title{
Preliminary Simulation and Design of a Weight In Motion System
}

\author{
Andrea Rindi, Luca Pugi, Enrico Meli \\ Università degli Studi di Firenze, Dip. Energetica Sergio Stecco
}

\begin{abstract}
Aim of WIM Systems (Weight In Motion) is to evaluate the loading conditions (axle/wheel weight) of a railway vehicle in motion from the measurement of induced stress-deformations on railway infrastructure (rails, sleepers, etc.).

This kind of measurements are quite important for safety and maintenance purposes in order to verify the loading conditions of a wide population of vehicles using a limited number of WIM devices distributed on the rail network.

The evaluation of axle loading conditions is quite important especially for freight wagons, more subjected to the risk of unbalanced loads which may be extremely dangerous both for vehicle safety and infrastructure maintenance costs.

Also some typical defects of rolling surfaces such as wheel flat should be identified especially by considering frequency and time domain analysis or mixed/hybrid approaches as wavelet transformations.

In this work a simplified FEM model of vehicle-rail interaction is used to investigate potential performances and robustness of a proposed WIM algorithms against disturbances, bandwidth/resolution limitations and sensors and estimators bias errors.
\end{abstract}

Keywords: Weight In Motion, Finite Element Methods, Axle Load identification.

\section{Introduction and state of the art}

The development of efficient WIM (Weight In Motion) systems is quite interesting both from an industrial and academic point of view. Aim of a WIM system is the estimation/weighting of axle of vehicles in motion.

In Figure 1 some examples of measurements used for WIM application are shown: 
- Shear Stress/deformation measurements on rail[1],[2],[3]: a circular notch/slot is drilled over the rail web on the mean-neutral line where approximately pure shear stresses are applied. Stress-Deformations concentrations arising in the notch are measured using conventional strain gage sensors or piezoelectric/piezo-resistive systems to estimate rail shear efforts and consequently the vertical force due to axle load..

- Rail Bending [4],[5]: Strain sensitive elements are used to evaluate deformations due to bending/shear typically on the rail foot. Contactless measurements of displacements may be also performed using Laser Doppler Sensors.

- Sensorized Sleepers: force sensitive elements are placed over the sleepers in the section corresponding to rail baseplate/pads. Also in this case deformation/stress over a deformable spring elements are measured.

- Sensitive Bridge/SlabTrack Systems: Sleepers/rails are constrained over an instrumented structure which works as spring elements to measure vertical axle loads.

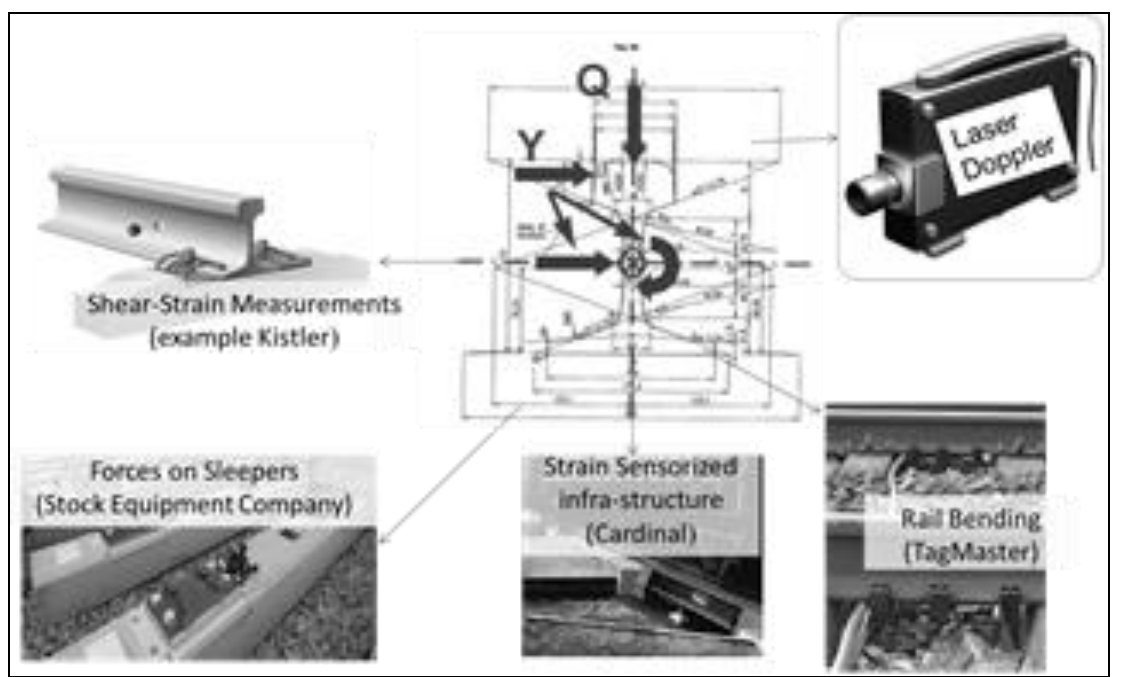

Figure 1: Forces acting on a rail section and examples of different measurement techniques

WIM Systems based on rail stress/deformation measurements are the most diffused mainly since they usually assure higher performances in terms of bandwidth, precision, linearity. Also respect to other measurement solution are less affected by different construction methods of the infrastructure (as for example, ballasted or slab track).

Both bending or shear measurements on the rail may be optimized in order to reject as much as possible spurious signals due to lateral forces ( $\mathrm{Y}$ in Figure 1) or to longitudinal tensile components due to thermally induced coaction loads.

Lateral dynamic of the vehicle may also produce load transfers between left and right wheels of the same axle. In order to measure the total load on each axle force/strain measurements coming from left and right of the same measurement sections should be summed as visible in the simplified scheme of Figure 2. 
Some additional information concerning vehicle speed, geometrical or inertial properties should be identified using additional sensors or transmitted by the vehicle itself using low cost technologies like RFID (Radio Frequency IDentification).

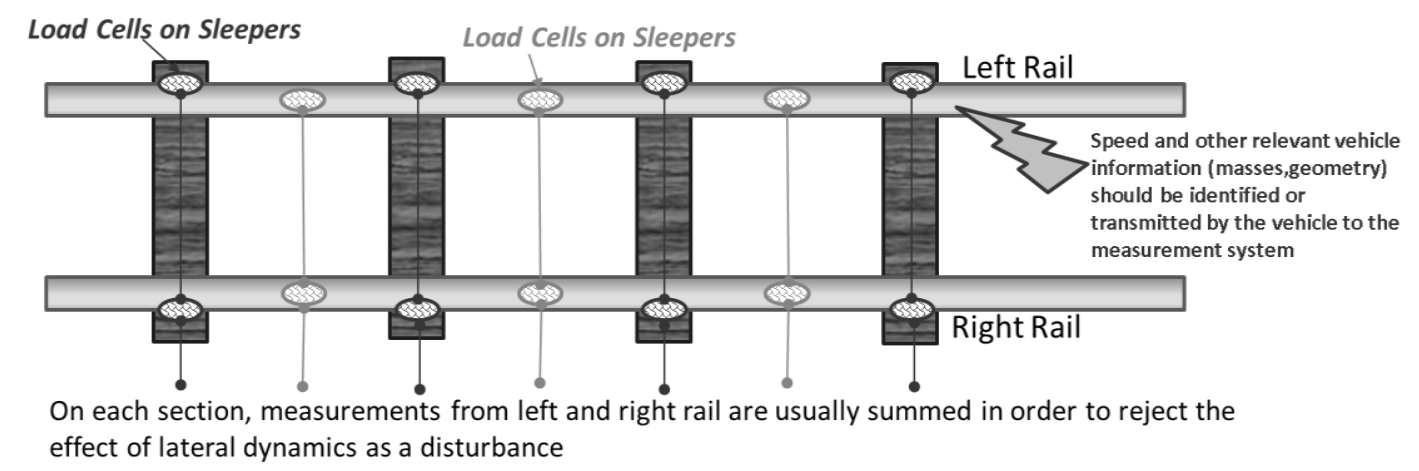

Figure 2: example of simplified layout

Since the measurement layout is designed to reject the effects of lateral dynamics as disturbances, all the system is often studied using simplified planar models of both vehicle and infrastructure as visible in the example of Figure 3: a lumped planar model of the vehicle is implemented using a system of ordinary differential equations (ODE); rail is modelled as a continuous beam (using FEM or other PDE discretizing methods) constrained by discrete visco-elastic elements which represents the pinned supports of sleepers on ballast. A simplified model (for example a lumped spring or an imposed motion constraint) is used to simulate wheel-rail contact coupling vehicle and rail models.

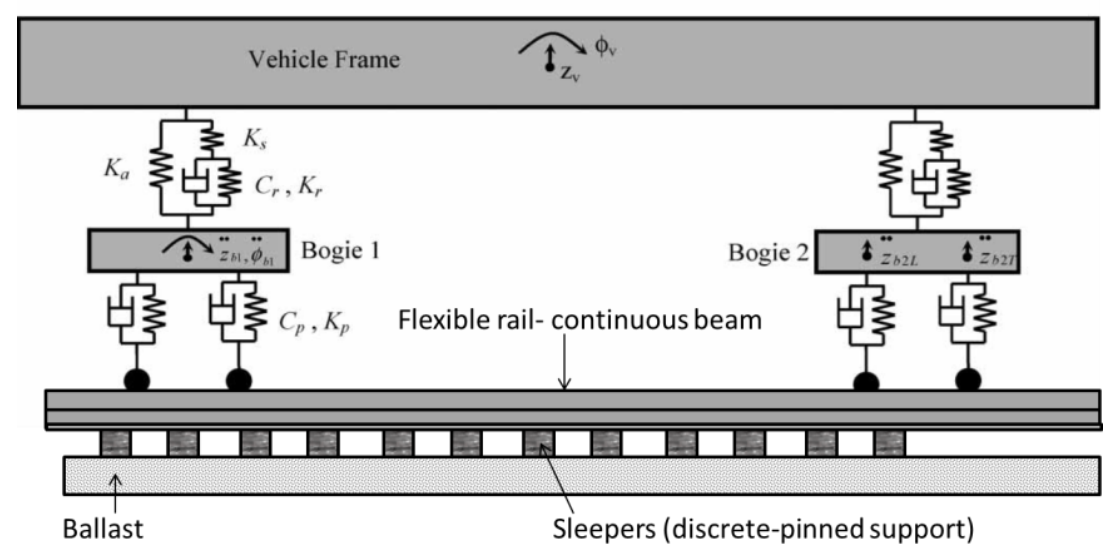

Figure 3: Example of planar vehicle-infrastructure model used to simulate wheel rail interaction

In this work, simplified models as the one showed in Figure 3, are used to produce synthetic inputs that are used to virtually train, calibrate and verify the performance of a proposed/simulated WIM system as stated by the flowchart of Figure 4 . 


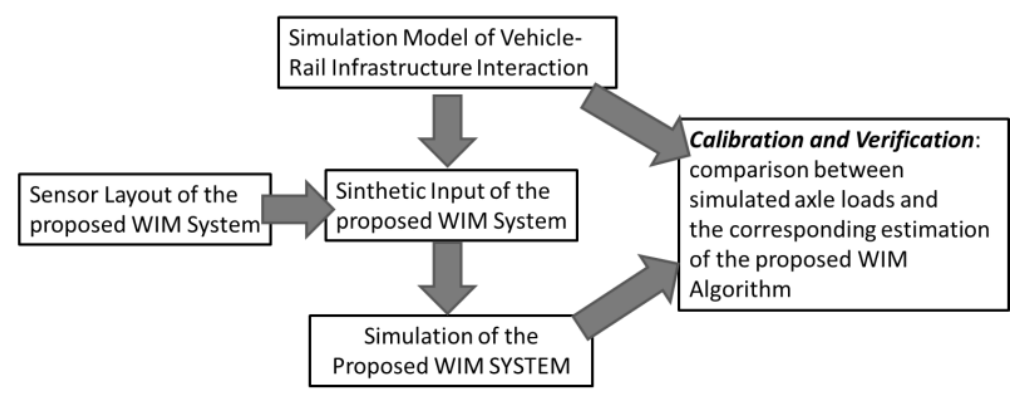

Figure 4: Development, calibration and verification of WIM system using simulation models

\section{Modelling of the rail-infrastructure: model used for the calibration of the proposed WIM system}

Rail and the underlying infrastructure are modelled as a continuous beam representing the rail supported by an elastic foundation which simulates sleepers, ballast and the underlying infrastructure. For the rail both Eulero-Bernoulli and Timoshenko models can be used. In particular the Eulero-Bernoulli beam model neglects the shear deformability considering only the contribution of bending (1):

$E I \frac{\partial^{4} v(x, t)}{\partial x^{4}}+\rho A \frac{\partial^{2} v(x, t)}{\partial t^{2}}=q(x, t)$

$E, \rho=$ Young modulus and density of the material

$A, I=$ Area and momentum of the beam section

$x, t=$ longitudinal position and time

$q, v=$ distributed load and vertical displacement.

The contribution of shear deformability mainly affects higher frequency modes where the Eulero-Bernoulli model is more likely to over-estimate the corresponding eigen-frequencies. From bibliography [6] a typical benchmark case studied is considered: a "free" (not constrained) rail of 4 meters is simulated using different models of beam and performing the calculation of eigenmodes and eigenfrequencies. As visible in Table 1 Eulero-Bernoulli introduces an overestimation of high frequency modes associated to low spatial scales. In the considered example, overestimation for frequency beyond $500 \mathrm{~Hz}$ introduces severe errors. 


\begin{tabular}{|c|c|c|c|c|}
\hline $\begin{array}{c}\text { Eigen Frequencies } \\
\text { (Experimental) }\end{array}$ & $\begin{array}{c}\text { Calculated (Eulero - } \\
\text { Bernoulli Model) }\end{array}$ & $\begin{array}{c}\text { Calculated (Raleigh } \\
- \text { Timoshenko Model, } \\
\boldsymbol{k}=\mathbf{0 . 3 4 )}\end{array}$ & $\begin{array}{c}\text { Calculated (Raleigh } \\
- \text { Timoshenko Model, } \\
\boldsymbol{k}=\mathbf{0 . 3 8})\end{array}$ & $\begin{array}{c}\text { Calculated (Raleigh } \\
- \text { Timoshenko Model, } \\
\boldsymbol{k}=\mathbf{0 . 4 0 )}\end{array}$ \\
\hline $64 \mathrm{~Hz}$ & $63.5 \mathrm{~Hz}$ & $64.8 \mathrm{~Hz}$ & $64.9 \mathrm{~Hz}$ & $64.9 \mathrm{~Hz}$ \\
\hline $174 \mathrm{~Hz}$ & $175 \mathrm{~Hz}$ & $173 \mathrm{~Hz}$ & $173 \mathrm{~Hz}$ & $174 \mathrm{~Hz}$ \\
\hline $327 \mathrm{~Hz}$ & $343 \mathrm{~Hz}$ & $324 \mathrm{~Hz}$ & $326 \mathrm{~Hz}$ & $327 \mathrm{~Hz}$ \\
\hline \multicolumn{4}{|r|}{} & $\begin{array}{c}\text { K is the shear factor } \\
\text { for the Raleigh } \\
\text { Timoshenko Beam }\end{array}$ \\
\hline
\end{tabular}

Table 1: Calculation of rail eigenmodes considering different beam models

Since the bandwidth of the WIM algorithm proposed in this work is far lower than $100 \mathrm{~Hz}$, authors prefer to use Eulero-Bernoulli models to simulate the rails, also considering that higher frequency modes are heavily affected by damping and by other non-linear phenomena that have to be calibrated on experimental data [6],[7],[10],[12],[13],[15],[16].

In particular in this work the structural damping of the rail is modelled using the socalled "proportional" or "Raileigh" damping: the damping matrix $[C]$ is calculated as a linear combination of stiffness $[K]$ and inertia $[M]$ matrices of the structure according to(2):

$[C]=\alpha[M]+\beta[K]$

$\alpha, \beta=$ constant proportionality factors.

The coefficients $\alpha$ and $\beta$ are calibrated in order to fit the typical behaviour which is expected from results and physical considerations available in bibliography[6], [10], [16].

In order to model the contribution of the deformability of sleepers and ballast different models of increasing complexity may be adopted.

One of the simplest formulations may be the so called "Winkler continuous supported beam" [7] that models ballast and sleepers as a continuous visco-elastic soil to which the rail is constrained, as visible in the simplified scheme of Figure 5. The Winkler model neglects the discrete nature of the sleeper constraints introducing approximation that are too large for the study of a WIM system.

\section{Continuous Beam(Bernoulli or Timoshenko)}

\section{Winkler continuous supportifoundation}

Figure 5: Beam model with continuous support foundation

To consider the discrete nature of the sleeper constraint, the sleepers have to be modelled as lumped systems of springs, masses and dampers which try to reproduce/fit the modal behaviour of the infrastructure. Increasing the number of degrees of freedom used to model a sleeper usually leads to an improvement of the model accuracy. In particular one of the most accurate models is introduced by Dahlberg [6][10]: each sleeper is modelled with a two degrees of freedom lumped system in order to introduce at least two suspension stages with corresponding 
poles/eigenfrequencies. Also ballast exhibits a non-linear damping and residual/cumulative deformations; so Coulomb friction elements or series/parallel combinations of spring and dampers may be introduced in the model as visible in the scheme of Figure 6.

Also commercial multibody software, mainly used for ride stability and comfort of railway vehicles, often use the two stage suspension approach to model ballast and sleeper flexibility (as shown in the example of Figure 7) where additional degrees of freedom due to the rotation of the structure are introduced to simulate the threedimensional behaviour of the line.

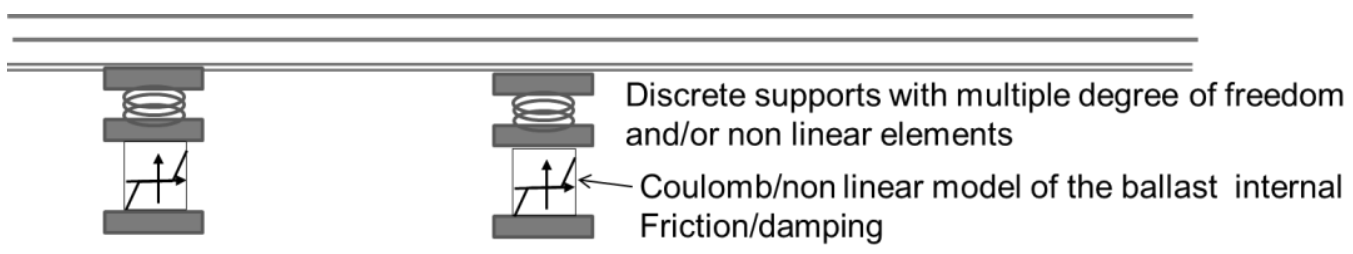

Figure 6: Continuous beam model on discrete supports; ballast modelled with non-linear spring friction elements

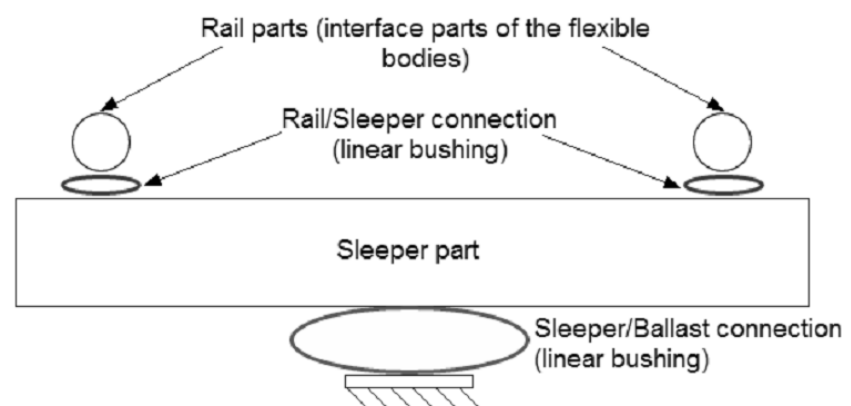

Figure 7: Flexible model used by a commercial multibody software [11]

For the simulation and the validation of the proposed WIM system author preferred to use the simplified model proposed by Kisilowksy [8] which is often used in literature for the simulation and the development of this kind of applications[1],[2], [9],[14]. In the proposed model, visible in Figure 8, sleepers are simulated as single degree of freedom system resulting a good compromise between accuracy and simplicity.

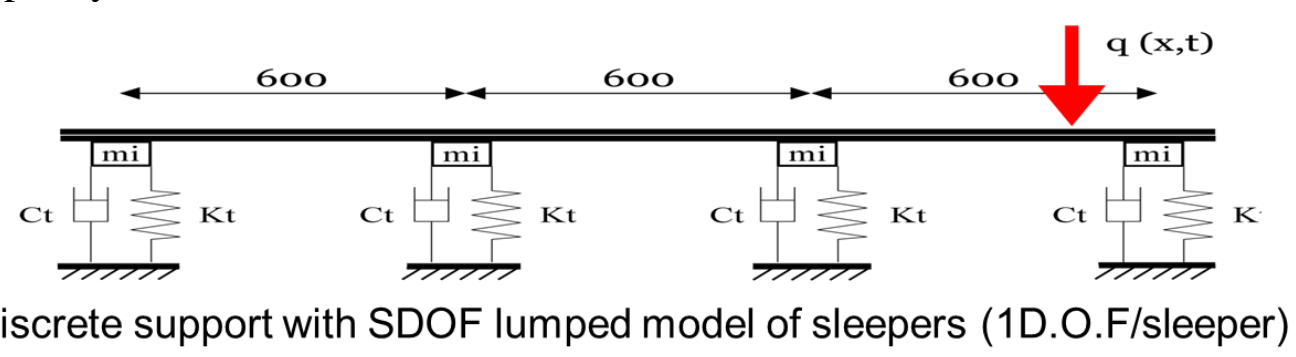

Figure 8: Continuous beam model on discrete supports (1 vertical degree of Freedom per sleeper) 
In particular the FEM model used for this study has been developed using ComsolMultiphisics $^{\mathrm{TM}}$; so all the parameters can be parametrically edited using MathworksMatlab ${ }^{\mathrm{TM}}$ which have been intensively used for pre-processing, post-processing and for the simulation of the proposed WIM algorithm.

Main features of the proposed model of the railway line are visible in Table 2.

\begin{tabular}{|c||c|c||c|}
\hline Simulated rail & UIC 60 & $\boldsymbol{M i}$ & $10 \mathrm{Kg}$ \\
\hline Young modulus & $20000 \mathrm{~N} / \mathrm{mm}^{2}$ & $\boldsymbol{C t}$ & $250000 \mathrm{Ns} / \mathrm{m}$ \\
\hline Struct. damping $\boldsymbol{\alpha}$ & $301 / \mathrm{s}$ & $\boldsymbol{K} \boldsymbol{t}$ & $50000000 \mathrm{~N} / \mathrm{m}$ \\
\hline Struct. damping $\boldsymbol{\beta}$ & $0.003 \mathrm{~s}$ & $\begin{array}{c}\text { Distance between } \\
\text { sleepers }\end{array}$ & $0.6 \mathrm{~m}$ \\
\hline
\end{tabular}

Table 2: Physical data used for the simulation of the railway line

In the proposed model additional features can be modelled in order to increase model accuracy or even to test robustness against tolerances and modelling errors:

- additional stiffness, damping and inertia elements against rail rotation

- customizable non-linear behaviour of each damping and stiffness lumped element.

\section{Vehicle Modelling}

The rail-infrastructure model described in the previous chapters has to interact with a simplified model of the traveling vertical loads introduced by the vehicle axles. Since the model adopted for the railway line is planar also a bi-dimensional approach has to be followed for the vehicle, completely neglecting the contribution of the lateral dynamics.

Since different models of the travelling axle loads can influence simulation results, authors have evaluated and compared results obtained from different models of the vehicle:

Traveling force: a lumped force translating at the same speed of the vehicle as visible in

a) Figure 9

Traveling force with axle: the unsprang masse of the axle and the force contribution of the axle inertia are considered as visible in

b) Figure 9

Quarter vehicle model: a single stage suspension system is considered as visible in

c) Figure 9

Quarter vehicle model: also an additional D.O.F. introduced by the vertical stiffness and damping of the wheel - rail contact is considered as visible in

d) Figure 9

A complete model of a railway vehicle with a two stage suspension system and a B-B wheelset (according to the simplified scheme also used by Esveld [7]) is considered to simulate vehicle-line interaction as visible in

e) Figure 10 . 
For all the the above mentioned models, inertial and suspension properties are inspired to Italian Shimns freight wagons whose main features have been taken from a literature[17]. For the model "e)" also data of a known Italian passenger coach[18] have been used to perform simulations.

b)

a)

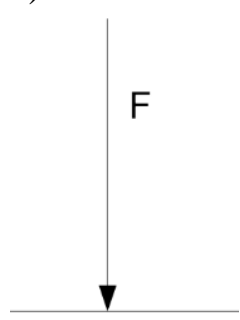

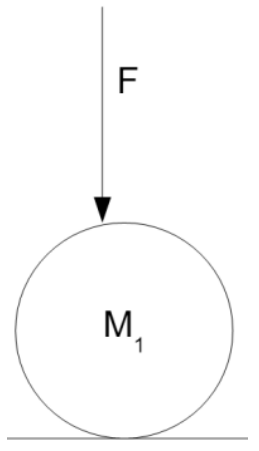

c)

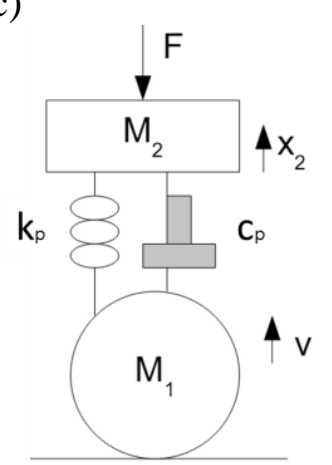

d)

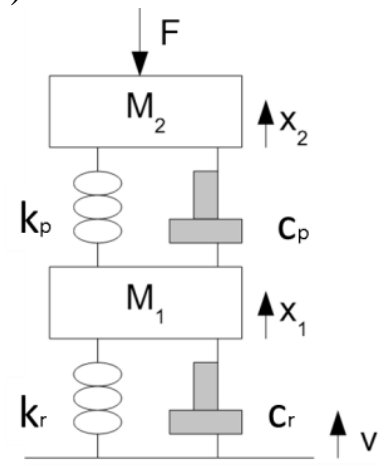

Figure 9: Simplified models used to simulate the axle loads (bracketed letters indicate the corresponding model)

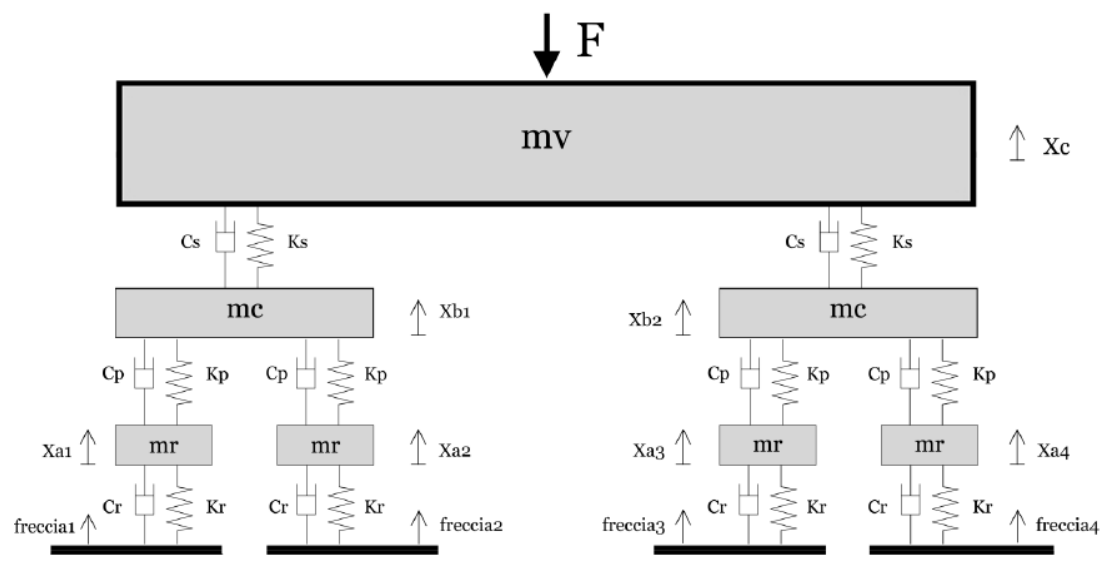

Figure 10: Planar vehicle model

In Figure 11, the calculated bending stress in the bottom fibre of a rail due to a single axle traveling at $18 \mathrm{~m} / \mathrm{s}$ (about $65 \mathrm{~km} / \mathrm{h}$ ) is shown: simulation is repeated using different axle models showing very little differences in results. As a consequence, neglecting the effect of irregularities on rolling surfaces, it is possible to affirm that simulation results are quite robust against vehicle parameter uncertainties or modelling error. 


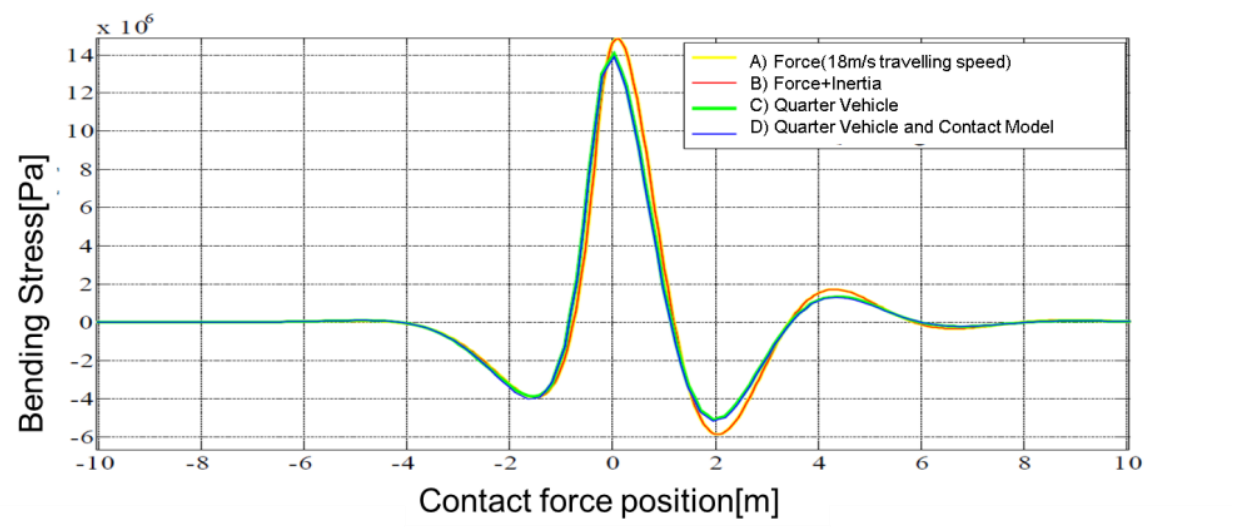

Figure 11: Simulated bending stress at the bottom fibre of the rail calculated considering different axle load models

The same simulation is repeated comparing the results obtained with the planar model vehicle "e)" and a sum of traveling forces corresponding to the model "a)" applied with a delay that reproduces the relative positioning of axles and bogies along the vehicle.

The corresponding results in terms of calculated bending stress on the bottom fibre of the rail are shown in Figure 12 : also in this case the differences in results are quite small, near to negligible. As a consequence the effect of a vehicle traveling on the line at a known speed is almost equivalent to the application of the traveling forces corresponding to the loads of the single axles as visible in Figure 12. Considering the linearity of the load a) and of the proposed model of the line, the principle of superposition of effects can be applied: the solution corresponding to the application of the multiple traveling loads relative to the considered vehicle (model e)) is equivalent to the sum of the effects of the single forces (model a)). This result, which is only approximately true, it is very important for the development of a WIM estimation algorithm since the observed solution due the passing of a vehicle can be approximately decomposed as the sum of the elementary solutions of the single axles. 


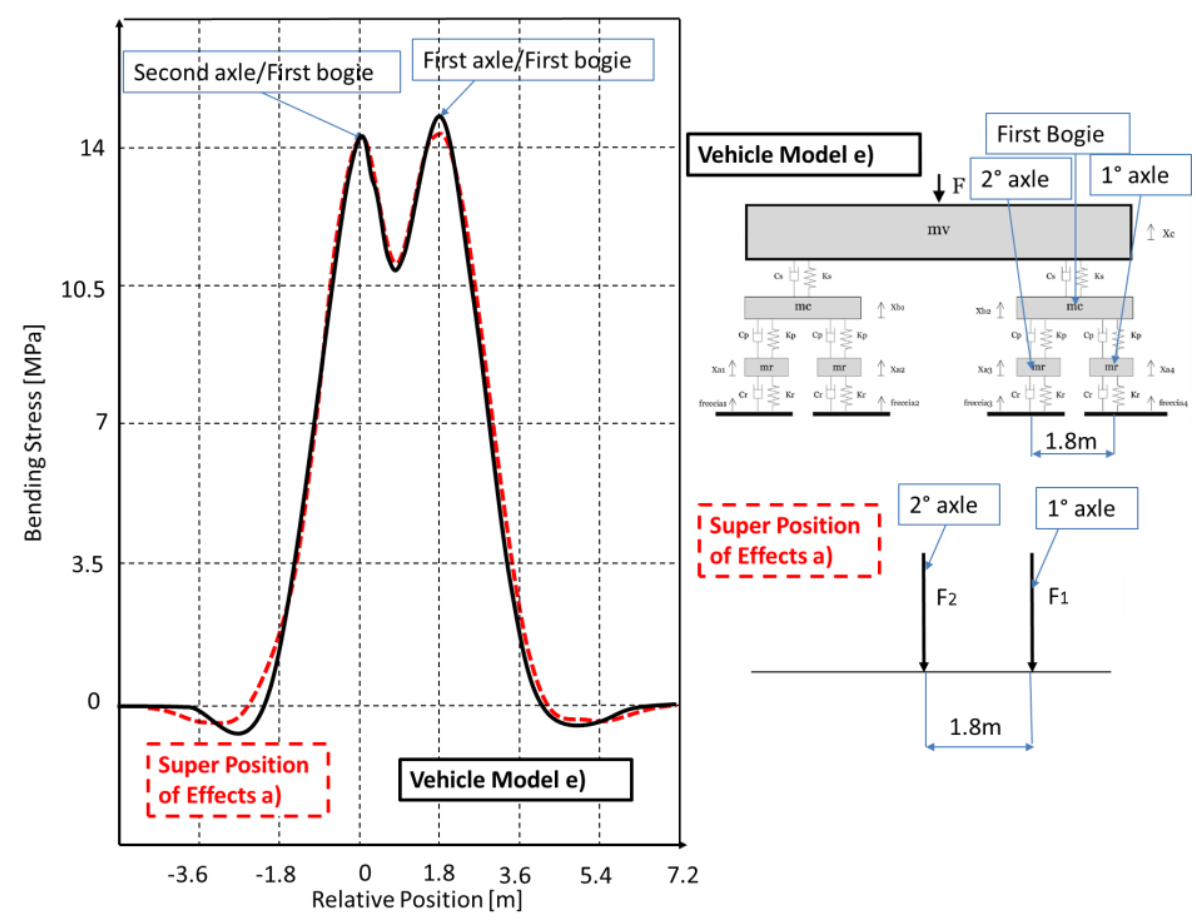

Figure 12: Simulated bending stress on the bottom fibre of the rail corresponding to the passage of two axle of a vehicle (model "e)") and of two traveling forces $F_{1}, F_{2}$ with equivalent loads

According to the superposition of effects, let $f(x, t)=\mathfrak{I}(F)$ a physical quantity measured on the track (stress, deformations, forces, etc.) corresponding to the application of a force $F(x, t)=\delta\left(x-x_{0}-v\left(t-t_{0}\right)\right) \quad(\delta$ is the Dirac delta function) traveling at constant speed $v$ with an unitary modulus.

A generic force $F^{*}=a \delta\left(x-x_{0}-v\left(t-t_{d}-t_{0}\right)\right)$ traveling at speed $v$ with an arbitrary modulus $a$ and delayed of $t_{d}$ with respect to $F$ can be defined as (3):

$F^{*}(x, t)=a F\left(x, t-t_{d}\right)$.

As a consequence, the solution $f^{*}$ corresponding to the application of $F^{*}$ can be easily calculated starting from $f$ :

$f^{*}(x, t)=\mathfrak{I}\left(F^{*}\right)=a f\left(x, t-t_{d}\right)$.

When $n$ arbitrary forces $F_{i}=a_{i} \delta\left(x-x_{0}-v\left(t-t_{d i}-t_{0}\right)\right) \quad(i=1,2, \ldots n)$ with modulus $a_{i}$ and delay $t_{d i}$ traveling at same speed $v$ are applied, the corresponding solution $f_{\text {tot }}$ can be calculated as follows (5):

$f_{\text {tot }}=\mathfrak{J}\left(\sum_{i=1}^{n} F_{i}\right)=\sum_{i=1}^{n} a_{i} f\left(x, t-t_{d i}\right)$ 
Since the measurements are performed at different temporal instants $t_{k}$ $(k=1,2, \ldots m)$ and by means of different sensors (with longitudinal position $x_{j}$, $j=1,2, \ldots p$ ), equation (5) can be rewritten in a more general form:

$f_{\text {tot }}^{j k}=\mathfrak{I}\left(\sum_{i=1}^{n} F_{i}^{j k}\right)=\sum_{i=1}^{n} a_{i} f\left(x_{j}, t_{k}-t_{d i}\right)$.

\section{The Proposed WIM System}

Principle of operation of the proposed WIM system is described in the scheme of Figure 13 :

- Equation (5) can be written in the shape described by (7):

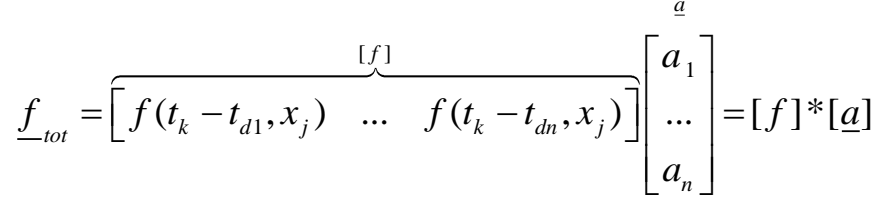

- The vector $\underline{f}_{\text {tot }} \in R^{m p}$ is supposed to be known and it is composed by a set of measurements that depends on the chosen sensors layout: for example a set of deformations measurements on different sections of the rails. Since real measurements are affected by noise and errors, numerical noise and errors (see equation (8)) have been also added to $\underline{f}_{t o t}$ :

$\underline{f}_{t o t}^{*}=\underline{f}_{t o t}+\underline{f}_{d}$

- The speed of the train $v$, the number of traveling axles $n$ and their relative positions are supposed to be known/measured by a dedicated estimator (and then, also in this case, affected by an error)

- The $n$ columns of the matrix $[f] \in R^{m p x n}$ are composed by the elementary solutions corresponding to the $n$ traveling forces

- of unitary modulus delayed by known delays $t_{i}$; using a reliable model of the rail-infrastructure it is possible to produce an estimation $\left[f^{*}\right]$ of $[f]$

- If the rank of $\left[f^{*}\right]$ is equal to $n$, it is possible to solve the system (9) where the scalar coefficients $a_{i}^{*}$ represent the estimated values of the axle loads:

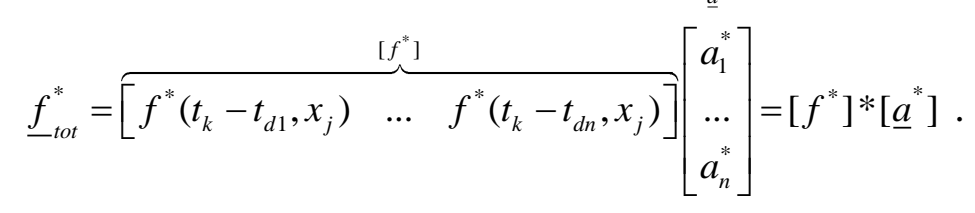




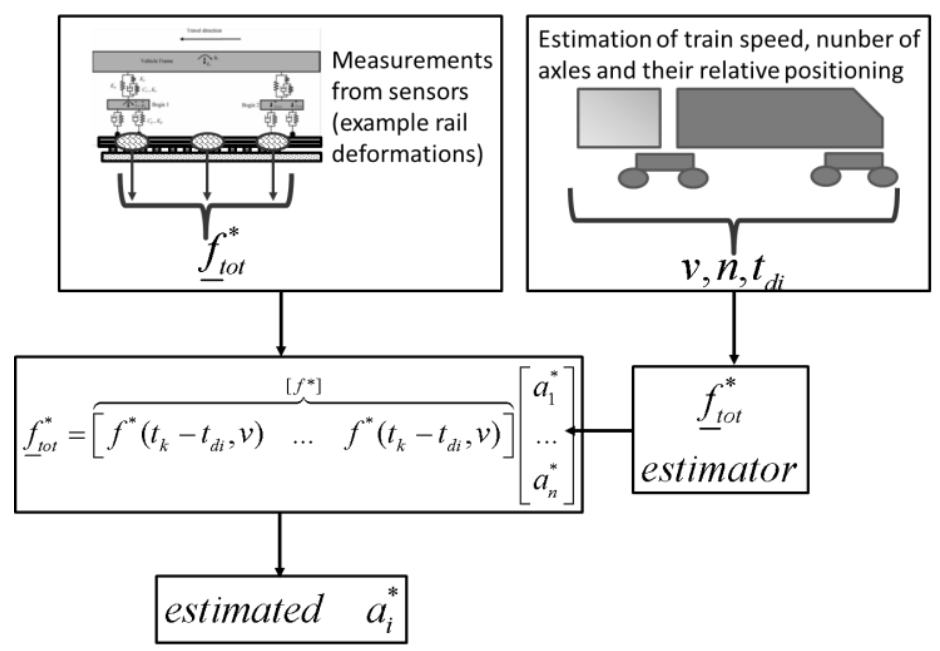

Figure 13: Proposed WIM system, principle of operation

In order to reduce the errors introduced by disturbances, it is convenient to use an high number of measurements; so the size $m p$ of the vector $\underline{f}_{\text {tot }}^{*}$ and consequently the number of scalar equations corresponding to system (9) is far higher than $n$.

As a consequence the calculated solution will be an approximation of the experimental data calculated according to a chosen best fit criteria which will depend on the adopted solving method.

By simply calculating the pseudo-inverse of $\left[f^{*}\right]$, it is possible to evaluate the solution $\underline{a}^{*}$ in terms of least square best fit, as proposed in this work:

$$
\underline{a}^{*}=\left(\left[f^{*}\right]^{T}\left[f^{*}\right]\right)^{-1}\left[f^{*}\right]^{T} \underline{f}_{t o t}^{*} .
$$

\subsection{Measurements on the Rail Infrastructure}

The proposed approach is applicable to different sensor layouts/measurements as in the examples described in the introduction of this work.

The chosen sensor layout mainly affects some critical aspects:

- Signal to noise ratio: errors and noise on signal measurements may negatively affect the measurement $\underline{f}_{t o t}^{*}$ with respect to the real values of $\underline{f}_{t o t}$, introducing errors in the estimation

- Bandwidth: bandwidth of the sensors used by the measurement system is necessary finite; therefore the bandwidth of the measured signals tends to increase with train speed; bandwidth of the sensors deeply influences the performances of the systems respect to train speed 
- Rank of $[f]$ : the chosen sensor-measurements affect the numerical conditioning of the matrix $[f]$ and consequently also of the estimated $\left[f^{*}\right]$.

- Finally the estimation of $\left[f^{*}\right]$ and the solution of system (9) involve the knowledge of $n, v, t_{d i}$ whose estimation depends also on the chosen sensor layout and is affected by errors

In the benchmark proposed in this work authors suppose that 10 adjacent sleepers of the instrumented railway line section are equipped with load cells or other sensors able to detect the vertical loads according to the scheme of Figure 14.

Information concerning $n, v, t_{d i}$ are supposed to be detected by a dedicated axle counter or radio transmitted by the passing train through a dedicated on board subsystem. A measurement section of 10 sleepers is considered a good compromise between accuracy and cost/reliability since, as visible in Fig. 11 e Fig. 15, the load corresponding to an axle is typically balanced by the reaction forces of the 5-7 nearest sleepers. The calculation is referred to a traveling speed of $5 \mathrm{~m} / \mathrm{s}-20 \mathrm{~m} / \mathrm{s}$ however this result seems to be quite insensitive to the value of the train speed.

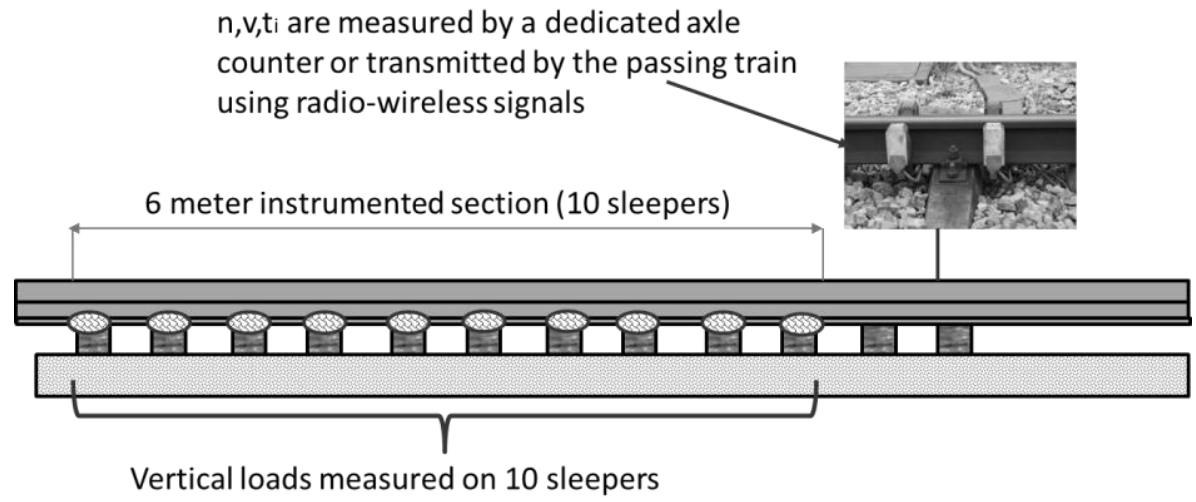

Figure 14: Proposed sensor layout

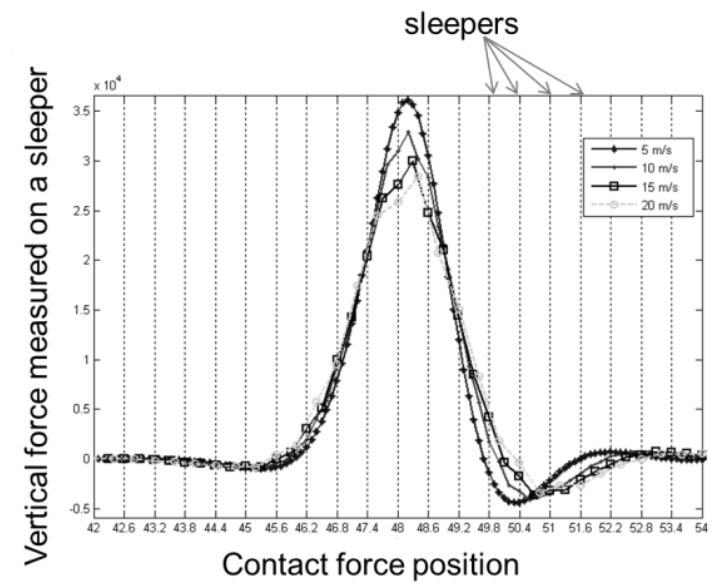

Shape of the solution change with speed but the number of affected sleeper/rail section is quite constant

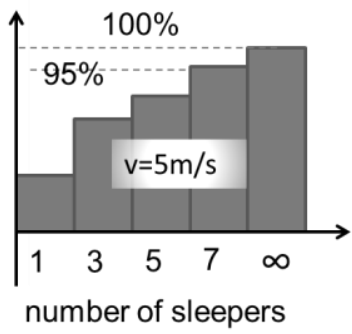

Figure 15: Cumulative load distribution on sleepers calculated for a train speed of $5 \mathrm{~m} / \mathrm{s}$ $20 \mathrm{~m} / \mathrm{s}$ 
Bandwidth of load cells inserted in the sleeper is modelled introducing a second order filter $G(s)$ described by (11) whose corresponding cutting frequency $\omega_{n}$ should be varied from 20 to $100 \mathrm{~Hz}$ to simulate cells with different bandwidths:

$G(s)=\frac{\omega_{n}^{2}}{s^{2}+2 \varepsilon \omega_{n} s+\omega_{n}^{2}}$.

White noise is added to the measured signal with an amplitude of about the 5\% of the supposed sensor range; finally a constant bias of about the $0.2 \%$ of the sensor range is applied to simulate a typical offset disturbance such an uncompensated thermal effect.

In this way it is possible to check the robustness of the algorithm against disturbances deriving from measurements.

\subsection{Robustness against modelling errors}

The estimated matrix $\left[f^{*}\right]$ is calculated trough a model of the railway line: in particular it is supposed the use of interpolated results coming from previously calculated FEM models. The model used to calculate $\left[f^{*}\right]$ may not be exact, since the response of a model is necessary an approximation of the response of the line corresponding to $[f]$.

As a consequence the matrix $[f]$ and the estimated $\left[f^{*}\right]$ are necessary different negatively affecting the precision of the WIM system.

In the proposed benchmark study the same FEM model has been considered both for simulating the railway line response and to calculate $\left[f^{*}\right]$ for the proposed WIM algorithm.

In order to verify the robustness of the proposed WIM system against modelling errors, the simulation of the railway line (used to generate the vector of measurements $\left.\left[f^{*}\right]\right)$ is deliberately perturbed modifying some simulation parameters like for example lumped stiffness, inertias and damping used to simulate sleepers or the structural damping coefficients $\alpha$ and $\beta$ of the rails.

In particular in this work the coefficients of each beam and sleeper element of the FEM model are randomly perturbed of about the $5-10 \%$ of their nominal value.

\section{Numerical Results}

In order to verify the accuracy of the proposed WIM system authors performed several simulations and benchmarks which generally confirm the reliability of the proposed approach. In particular, to verify the model ability of detecting complex configurations corresponding to multiple load, the functionality of the WIM system has been verified on a benchmark composition of two wagons with different axle loads visible in Figure 16. 


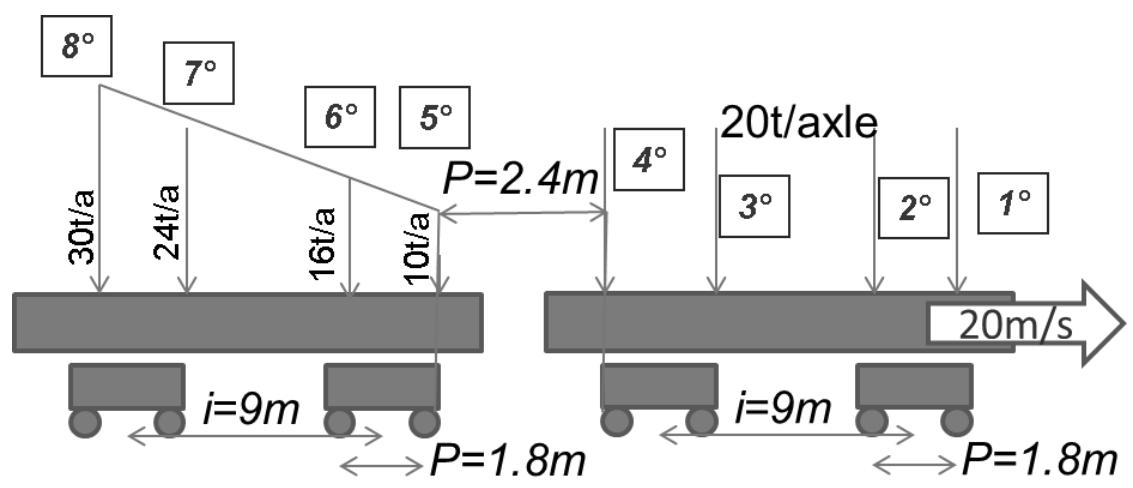

Figure 16: Benchmark composition used to test in simulation the proposed WIM system

The corresponding vertical force to which is subjected one of the instrumented sleepers of the WIM system is shown in Figure 17 : even considering a traveling speed of $20 \mathrm{~m} / \mathrm{s}(72 \mathrm{~km} / \mathrm{h})$ and a limited bandwidth of load cells of about $20 \mathrm{~Hz}$, the passage of each axle is clearly recognizable. The load of each axle is mainly distributed on the nearest 5-7 sleepers (3-4m); as a consequence there is a partially overlapping in the solution of the effects of two or three adjacent axles. This overlapping effect is evident for the passage of axle 5 which is less visible since the adjacent axles are more loaded.

In Table 3 relative errors on the axle load estimation performed by the proposed WIM algorithm are shown: even considering measurement noise, limited bandwidth and modelling errors previously described, the estimation error is limited to a 5-6\% while in optimal conditions (infinite bandwidth, no disturbance) is less than $1 \%$.

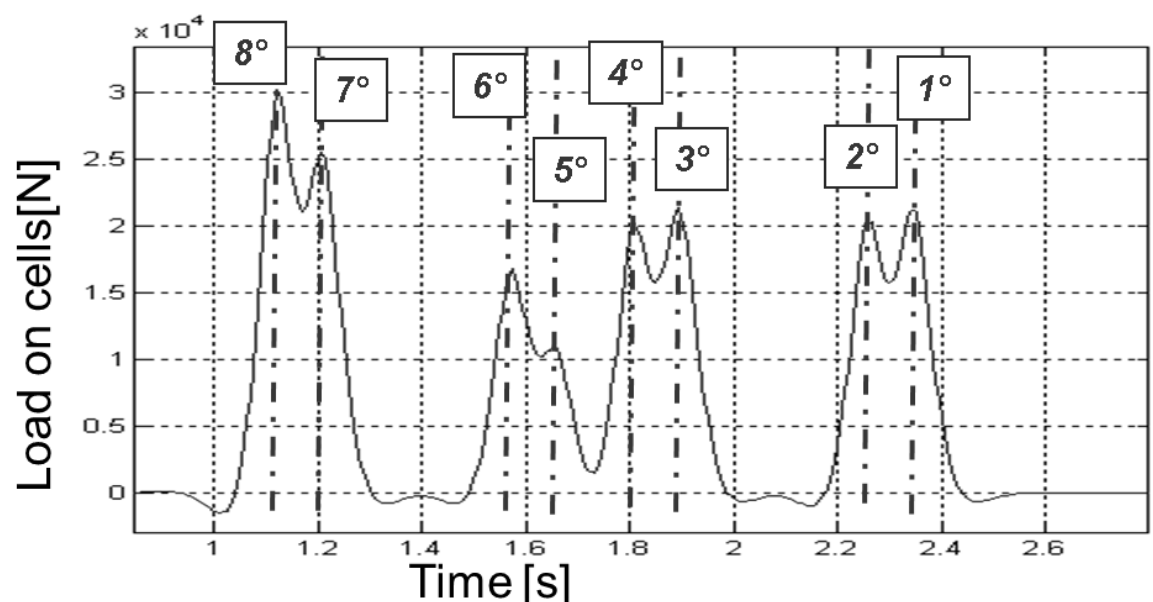

Figure 17: Force applied on a instrumented sleeper when the benchmark composition of Figure 16 is applied 


\begin{tabular}{|c|c||c|c|}
\hline Axle n. & Rel. Error \% & Axle n. & Rel. Error \% \\
\hline $1^{\circ}$ & $5.2 \%$ & $5^{\circ}$ & $2.6 \%$ \\
\hline $2^{\circ}$ & $4.5 \%$ & $6^{\circ}$ & $6.5 \%$ \\
\hline $3^{\circ}$ & $5.4 \%$ & $7^{\circ}$ & $4.6 \%$ \\
\hline $4^{\circ}$ & $3.9 \%$ & $8^{\circ}$ & $5.1 \%$ \\
\hline
\end{tabular}

Table 3: Relative errors in axle load estimation (disturbances applied)

Results of more general interest may be obtained, performing multiple scenario, in which the effect of the variability of some parameters is explored.

For example the same benchmark described in Figure 16 and Figure 17 is repeated considering different traveling speed of vehicles and different performances of load cells in terms of bandwidth. The choice of bandwidth limitation is considered a good choice for a sensitivity analysis since a low bandwidth of the measurements may be also imposed by other fonts of noise or disturbances.

Some results concerning the maximum relative error in percentage respect to speed and bandwidth are visible in Figure 18: in the proposed benchmark the traveling axles are eight so the error shown is the maximum among the corresponding eight load estimations. It's interesting to notice that there is roughly linear relation between the available bandwidth of measurements and the corresponding traveling speed at which the load estimation became completely unreliable.

As a consequence further investigations considering experimental activities and real performances of the proposed sensor layout have to be considered mandatory for the future development of the research activities.

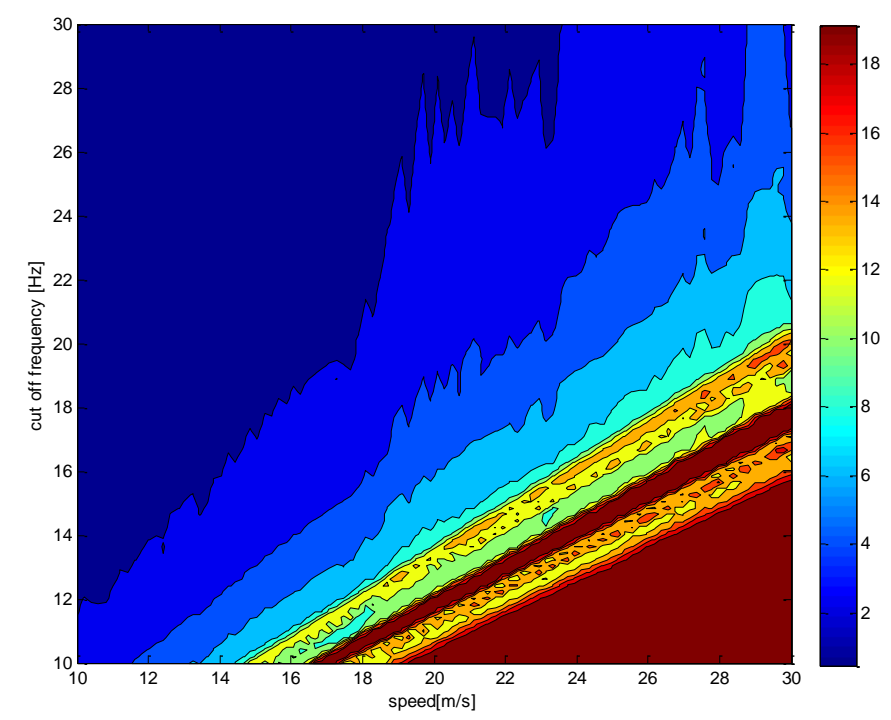

Figure 18: maximum relative error percentage as a function of vehicles traveling speed and measurements cut-off frequency 


\section{Conclusions and future developments}

In this work a general criteria for the design of a WIM system has been presented: a FEM model has been developed to simulate the necessary system inputs while the corresponding measurements are evaluated taking in account the limitations introduced by a near to realistic sensor system. The proposed algorithm is applied to an example of possible measurement layout simulating the effect of various sources of errors due to disturbances and to un-modelled dynamics. Preliminary results seems to be quite encouraging showing a general robustness of the proposed approach.

An interesting feature of the proposed approach is the extendibility of the method to different sensor/measurements layout.

Currently the work of the authors is focused on two different aspects:

- Optimization of the chosen sensor layout (number of channels, different typologies of measurements)

- Further optimization of WIM algorithm: currently the system performs a fitting based on the minimization of the squared norm of the error (least squares). A further optimization may investigate the possibility of further refining the fitting/identification criteria of the algorithm in order to reduce the dispersion of estimation errors between different axles

- Experimental activities: simulation results confirm that the chosen estimation algorithm is reliable; beyond further numerical optimizations of the algorithm experimental activities have to be performed in order to verify and optimize the system considering the real features of the proposed sensor layout.

Finally authors wish to thank all the students of the Engineering Faculty of Florence (Italy) that have stimulated this work with their fresh and enthusiastic approach.

\section{References}

[1] A. Bracciali R. Ciuffi, F. Piccioli "Progetto e validazione di un sensore estensimetrico multifunzionale per il binario ferroviario. Proceedings of $\mathrm{XXX}^{\circ}$ Congresso AIAS, Alghero, I, 2001

[2] C. del Prete C. Rosso An easy instrument and a methodology for the monitoring and the diagnosis of a rail Mechanical Systems and Signal Processing Volume 23, Issue 3, April 2009, Pages 940-956

[3] http://www.kistler.com/mediaaccess/200-580e-08.08.pdf technical doc. freely avaiable on the web

[4] http://www.gotchamonitoringsystems.com/pdf/Gotcha\%20Monitoring\%20Sys tems \%20-\%20System\%20Description.pdf technical doc. freely avaiable on the web

[5] P. Kołakowski, K. Sekuła, D. Sala, A. Swiercz \& A. Orłowska Weighing of trains in motion as a part of health monitoring system for a railway bridge, Proceding of Conference on Railway Engineering, London 2009 
[6] S. Iwnikcy, T. Dahlberg, - Track Issues - Handbook of railway vehicle dynamics,Cap.6, pp.143-178 - CRC Press Taylor and Francis Group, Boca Raton, FL, 2006

[7] Esveld C (1989): Modern railway track. MRT-Productions, Duisburg, Germany, ISBN 90-800324-1-7 (new edition 2001)

[8] J. Kisilowski - K. Knothe, Advanced railway vehicle-system dynamics, Warsaw, 1991

[9] A. Bracciali - R. Ciuffi - F. Piccioli, Soluzione numerica del modello PDE della dinamica verticale di un binario, Congresso AIAS 2001

[10] Dahlberg T (2001): Some railroad settlement models - a critical review, Proceedings of the Institution of Mechanical Engineers, Part F, Journal of Rail and Rapid Transit, Vol 215(F4), 289-300, 2001

[11] VI-Grade Technical Documentation ( VI-Rail 2005r2.11.0 Documentation)

[12] Denis M. Senyanskiy, PROBLEM OF INCREASING THE ACCURACY OF RAILWAY CARRIAGES WEIGHING IN MOTION VII IMEKO World Congress Metrology in the 3rd Millennium June 22-27, 2003, Dubrovnik, Croatia

[13] Ricardo Miguel da Costa Marques Pimentel, Maria Cristina Beirão Barbosa, Nuno Manuel Silva Costa,Diogo Rodrigo Ferreira Ribeiro, Luís Alberto de Almeida Ferreira, Francisco Manuel Moita Araújo, and Rui Artur Bártolo Calçada, Hybrid Fiber-Optic/Electrical Measurement System for Characterization of Railway Traffic and Its Effects on a Short Span Bridge IEEE SENSORS JOURNAL, VOL. 8, NO. 7, JULY 2008

[14] A. Bracciali, G. Cascini, M.Pieralli A Wheel Flat Detection Device based on Cepstrum Analysis of rail acceleration measurements

[15] A. Bracciali, F. Piccioli, "Attenuation of rail vibration: analysis of experimental data", Proc. WCRR 2001 World Congress on Railway Research, Köhln, DE, 25-29 November 2001

[16] Jabbar Ali Zakeri, He Xia, Jun Jie Fan DYNAMIC RESPONSES OF TRAINTRACK SYSTEM TO SINGLE RAIL IRREGULARITY, Latin American Journal of Solids and Structures, Vol 6, No 2

[17] Luca Pugi ;Andrea Rindi; Andrea Giuseppe Ercole, Alessandro Palazzolo, Jury Auciello Duccio Fioravanti Mirko Ignesti, Preliminary studies concerning the application of different braking arrangements on Italian freight trains Vehicle System Dynamics: International Journal of Vehicle Mechanics and Mobility, 1744-5159, First published on 15 March 2011

[18] L.Pugi, M.Malvezzi A.Tarasconi,A.Palazzolo, G.Cocci,M.Violani Simulation of WSP Systems on MI-6 Test rig, Vehicle system and dynamics ( ed.Taylor and Francis) Vol. 44, Supplement 2006 pp.843-852 ISBN 978-0-41543616-8 